\title{
Influence of Monomer Sequence of Binder Polymers for Lithographic Property of Photodefinable Nanocomposites
}

\author{
Yasuharu Murakami \\ Telecommunication Materials Development Center \\ Hitachi Chemical Co., Ltd. Tsukuba Research Laboratory, \\ 4-13-1 Higashi-cho, Hitachi, Ibaraki, 317-8555, Japan
}

\begin{abstract}
The relationship of binder polymer compositions and lithographic properties was examined and following conclusions were obtained; (1) The alternating sequence of binder polymer is preferable to the nanocomposite of excellent transparency. (2) The moiety of methacrylic acid is necessary to be $50 \%$ or more for the residue-free patterning. (3) Photo-definable nanocomposite composed of alternating copolymer and nano titania gives a patternable nanocomposite with high refractive index.
\end{abstract}

Keyword: photopolymer, binderpolymer, sequence, nanocomposite

\section{Introduction}

Photodefinable nanocomposites (PDNCs), which are nano-scale composites of photodefinable polymer with nanoparticles, are used to improve mechanical, thermal, and optical properties of photodefinable polymers by choosing different kinds of nano-fillers[1-4]. There are three methods to prepare PDNCs, that is, sol-gel method, intercalation method, and the method of direct dispersion of nanoparticles. The sol-gel method has the disadvantages of shrinkage during reaction and poor uniformity of particles, although the transparence and the patterning properties of PDNC are sufficient. As for the intercalation and direct dispersion methods, they leave some residues after development when the volume of nanoparticles is high. However, these methods are advantageous in the simplicity of process and excellent dimensional accuracy of the obtained pattern.

In this research, we prepared and investigated the negative tone PDNCs of titania nanoparticles, cross-linkers, photoinitiators, and poly(styrene-comethacrylic acid) as a binder. We expected them to have excellent photodefinable properties even when used by the direct dispersion method. In the system, the copolymer seemed to act not only as a binder polymer, but also as a dispersant to avoid remaining residue after development. The relationships between the dispersibility of nano-fillers in the matrix and the photolithographic properties of PDNC were studied varying the types of binder polymers (different polymer sequence, component, molecular weight, and so on).

We believe that this fundamental research on photodefinable properties can apply not only to optical materials using titania nanoparticles, but also to other high performance materials using varios nanoparticles.

\section{Experimental}

\subsection{Materials}

Toluene (solvent parameter: $\mathrm{SP}=8.9$ ), $N, N$-dimethylformamide $\quad(\mathrm{DMF}, \quad \mathrm{SP}=12.0)$, $N$-methyl-2-pyrrolidone (NMP, $\mathrm{SP}=11.2$ ), ethylene glycol monomethyl ether (EGM, $\mathrm{SP}=11.5$ ), styrene, tert-butyl acrylate (TCI), methacroyl chloride (Tokyo Chemical Industry) and $p$-nitrophenol were supplied by Shanghai Chemical Reagent Company and used as received. $p$-Nitrophenyl methacrylate (NPMA) was synthesized [5] and purified by recrystallization 
from petroleum ether. $\mathrm{CuBr}$, Ethyl-2bromobutylate (EBB) and 2,2'-bipyridine (bpy) were supplied by Aldrich and used as received. 2,2'-Azobisisobutyronitrile (AIBN, Shanghai Chemical Reagent Company) was purified by recrystallization from metnanol. Nano titanium dioxides (first-order diameter: ca. $15 \mathrm{~nm}$, toluene/isopropanol dispersion (ca. $35 \mathrm{wt} \%$ )), HTD-884T (alumina/zirconia surface, with surfactant) and ND037 (polymer coating surface, surfactant free), were supplied from TAYCA Corporation (Table 1). Pentaerythritol triacrylate (PETA, Nippon Kayaku) and 2-benzyl-2-dimethylamino-1-(4-morpholino-phenyl)-butanone (Irg369, Ciba Specialty Chemicals) were used as received. Tetramethyl ammonium hydroxide (2.38 \%) (TMAH, Aldrich) was diluted ten times and used as a developer.

Table 1. Properties of nanofillers

\begin{tabular}{cccc}
\hline Nanofiller $^{* 1}$ & Surface treatment & $\begin{array}{c}\text { 1st-order } \\
\text { diameter }\end{array}$ & $\begin{array}{c}\text { 2nd-order } \\
\text { diameter }^{* 2}\end{array}$ \\
\hline HTD-884T & $\begin{array}{c}\text { alumina/zirconia } \\
\text { surface, with } \\
\text { surfactant } \\
\text { polymer coating } \\
\text { ND037 }\end{array}$ & $15 \mathrm{~nm}$ & $120 \mathrm{~nm}$ \\
& $\begin{array}{c}\text { surface, surfactant } \\
\text { free }\end{array}$ & $15 \mathrm{~nm}$ & $199 \mathrm{~nm}$ \\
\hline
\end{tabular}

*1 Toluene/isopropanol (1:1vol\%) dispersion (ca.35 wt\%). *2 Measured by particle size analyzer.

2.2. Synthesis of Polystyrene-alt-polyNPMA (PSt- $a$-PNPMA)

For the syntheses of polystyrene-altpolyacrylic acid and polystyrene-blockpolyacrylic acid, $p$-nitrophenol was used as a protecting group of acrylic acid. The group not only protects the acid part during block polymerization, but also controls electronic state of acrylic acid for alternating copolymerization.

In a shlenk tube with a magnetic bar, AIBN, styrene, NPMA, and toluene were added. The mixture was degassed three times under vacuum with liquid nitrogen. The polymerization was carried out at a certain temperature for a certain time. After the polymerization, the solution was poured into an excess amount of methanol, collected by filtration, and dried under vacuum. The polymer was purified by reprecipitation from acetone to methanol and dried under vacuum. The molecular weight was estimated by GPC measurement. The ${ }^{1} \mathrm{H}$ NMR measurements were conducted using acetone- $\mathrm{d}_{6}$ as a solvent. The ratio of styrene unit and NPMA unit in the resulting polymer was calculated according to the ratio of the intensity of signal from 5.8 to $7.4 \mathrm{ppm}$ (Aromatic $\mathrm{H}$ of styrene and NPMA) and that from 7.8 to $8.3 \mathrm{ppm}$ (Aromatic $\mathrm{H}$ of NPMA with alternating sequence).

\subsection{Synthesis of Macroinitiator}

In a shlenk tube with a magnetic bar, $\mathrm{CuBr}$ and bpy were mixed, after that, styrene, EBB, and toluene were added. Then, the polymerization and purification were carried out by the same procedure as the aforementioned case of PSt- $a$-PNPMA. The molecular weight was also estimated by GPC measurement.

\subsection{Synthesis of Polystyrene-block-polyNPMA (PSt- $b$-PNPMA)}

In a shlenk tube with a magnetic bar, $\mathrm{CuBr}$ and bpy were mixed, after that, NPMA, a macroinitiator, and a solvent were added. Then, PSt- $b$-PNPMA was obtained following the same procedure for PSt- $a$-PNPMA. The polymer was purified by reprecipitation from acetone to methanol and dried under vacuum. The molecular weight was estimated by GPC measurement. The ${ }^{1} \mathrm{H}$ NMR measurements were conducted using acetone- $d_{6}$ as a solvent. The ratio of styrene unit and NPMA unit in the resulting polymer was calculated according to the ratio of the intensity of signal from 6.4 to $7.6 \mathrm{ppm}$ (Aromatic $\mathrm{H}$ of styrene and NPMA) and that from 8.2 to $8.4 \mathrm{ppm}$ (Aromatic H of NPMA).

\subsection{Hydrolysis of Copolymers}

The copolymer was dissolved in THF. Potassium hydroxide in ethanol was added to the solution. The mixture was refluxed for $6 \mathrm{~h}$. The reaction mixture was poured into aqueous solution of hydrogen chloride. The precipitate adhered to the beaker. The precipitate was purified by reprecipitation from acetone to $n$-hexane and dried under vacuum.

\subsection{Synthesis of Polystyrene-random-poly methacrylic acid (PSt- $r$-PMAA)}

In a schlenk tube with a magnetic bar, AIBN, styrene (St), tert-butyl methacrylate (BMA), toluene and EGM were added. The mixture was degassed three times under vacuum with liquid nitrogen. The polymerization was carried out at $80{ }^{\circ} \mathrm{C}$ for $24 \mathrm{~h}$. After the polymerization, the solution was poured into an excess amount of methanol, collected by filtration, and dried under vacuum. The polymer was purified by reprecipitation from acetone to methanol 
and dried under vacuum. The polymer was obtained in $55 \%$ yield. The molecular weight was estimated by GPC measurement. The ${ }^{1} \mathrm{H}$ NMR measurements were conducted using DMSO- $d_{6}$ as a solvent. The copolymer was dissolved in $30 \mathrm{~mL}$ of dioxane. Hydrochloric acid was added to the solution. The mixture was refluxed for $6 \mathrm{~h}$. The reaction mixture was poured into aqueous solution of hydrogen chloride. The precipitate adhered to the beaker. The precipitate was purified by reprecipitation from acetone to $n$-hexane and dried under vacuum. The PSt- $r$-PMAA was yielded in $99 \%$. The other polymers were synthesized as reported procedure.

The values of $r_{1}(\mathrm{St})$ and $\mathrm{r}_{2}$ (BMA) were reported as 0.63 and 0.64, respectively [6]. The St/BMA sequence of $r_{1} r_{2}=0.403$, which indicates that the sequence is random. The properties of polymers are summarized in Table 2-4.

Table 2. Polymers with different monomer ratio

\begin{tabular}{ccccc}
\hline No & $\begin{array}{c}\text { St/MAA } \\
\text { sequence }^{* 1}\end{array}$ & St/MAA & $M_{\mathrm{w}}$ & $M_{\mathrm{w}} / M_{\mathrm{n}}$ \\
\hline 1 & Alternating & $33 / 66$ & 43,200 & 3.33 \\
2 & Alternating & $50 / 50$ & 23,600 & 1.96 \\
3 & Alternating & $56 / 44$ & 30,200 & 1.74 \\
4 & Alternating & $60 / 40$ & 20,600 & 1.71 \\
5 & Alternating & $65 / 35$ & 18,500 & 1.71 \\
6 & Alternating & $71 / 29$ & 17,000 & 1.73 \\
\hline *1 "Alternating" means alternating sequence of St/MAA. In \\
the case the feed ratio of monomer is not equivalent, the \\
excess amount of monomer forms homo-sequence unit.
\end{tabular}

Table 3. Polymers with different molecular weight

\begin{tabular}{ccccc} 
No & $\begin{array}{c}\text { St/MAA } \\
\text { sequence }\end{array}$ & St/MAA & $M_{\mathrm{w}}$ & $M_{\mathrm{w}} / M_{\mathrm{n}}$ \\
\hline 1 & Alternating & $57 / 43$ & 14,300 & 1.67 \\
2 & Alternating & $56 / 44$ & 30,200 & 1.74 \\
3 & Alternating & $55 / 45$ & 39,000 & 1.68 \\
\hline
\end{tabular}

Table 4. Polymers with different monomer sequence

\begin{tabular}{ccccc}
\hline No & $\begin{array}{c}\text { St/MAA } \\
\text { sequence }\end{array}$ & St/MAA & $M_{\mathrm{w}}$ & $M_{\mathrm{w}} / M_{\mathrm{n}}$ \\
\hline 1 & Alternating & $60 / 40$ & 20,600 & 1.71 \\
2 & Random & $60 / 40$ & 25,200 & 1.46 \\
3 & Block & $60 / 40$ & 8,600 & 1.39 \\
\hline
\end{tabular}

2.7. Preparation of Photo-definable Nanocomposites (PDNCs)

Certain amounts of the binder polymers (PSt- $r$-PMAA or PSt- $b$-PMAA), PETA, titania, and Irg369 were placed and mixed in a plastic bottle. Then, the mixture was applied on silicon wafer. By being dried in oven at $110{ }^{\circ} \mathrm{C}$ for $10 \mathrm{~min}$,
PDNC layer was formed on the wafer.

\subsection{Transparency of PDNC}

The transparency of PDNC was estimated by measuring transmittance using UV-vis spectroscopy. The sample was prepared on the glass plate by spincoating and baking in an oven at $100{ }^{\circ} \mathrm{C}$ for $5 \mathrm{~min}$. The transmittances of each sample were normalized as $10 \mu \mathrm{m}$ thickness and compared at $600 \mathrm{~nm}$.

\subsection{Photolithography}

The PDNC was exposed by use of an exposure equipment with high pressure mercury lamp through glass photomask. The exposed samples were developed by dipping into $1 \%$ tetramethyl ammonium hydroxide (TMAH) at $30{ }^{\circ} \mathrm{C}$ to afford the patterned nanocomposite.

\section{Results and Discussion}

3.1. Transparency of PDNC and binder polymer composition

Figure 1 shows the transmittances of PDNC with different kinds of binder polymer which has different sequence. The measured PDNCs were composed of binder polymer (PSt-co-PMAA, St:MAA $=60: 40$ ), photocrosslinkable monomer (PETA), and nano-titania filler (HTD-884T).

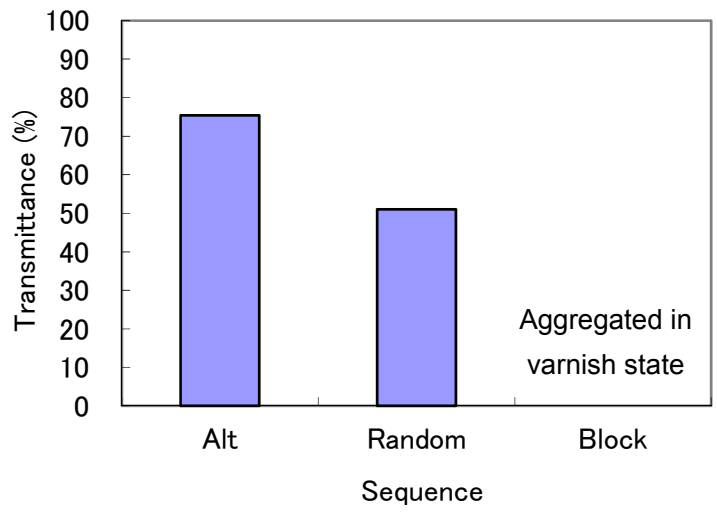

Figure 1. Polymer sequence and transmittance of PDNC.

It was found that the PDNC contained binder polymer with alternating sequence showed higher transmittance than that with random. In the case of block polymer, the filler was aggregated and the uniform film could not be formed on the glass substrate.

These results show that the high content of alternating sequence increases transmittance of PDNC. Two reasons are considered for the 
difference of transparency. First, the continuous domain causes a phase separation to induce the aggregation of nanoparticles in the matrix. Second, the coordination strength of polymers to the nanoparticles was increased by more condensed carboxylic acid domains. The coordination might destroy the dispersion of nanoparticles. We assumed that the second reason would be more acceptable, because the aggregation had already been observed in varnish state with block polymer system, in which phase separation did not occur yet.

Figure 2 shows the relationship between methacrylic acid (MAA) contents and transmittances of PDNCs with different kinds of titania nano-particles (the surfaces are either alumina or polymer). For the alumina surface particles, the transmittance increased with increases in the MAA content. The transmittance was saturated at around $80 \%$, with over $40 \%$ MAA content. In the polymer coated titania nano-particle system, the transmittance remained stable at around $80 \%$, whereas the PDNC with the polymer coated nano-particles was insoluble in an alkaline solution. On the basis of these results, we concluded that the use of (1) the binder polymer with over 40\% MAA contents and (2) the alumina surface nano-particles stabilized by surfactant, are both required for the alkaline developable PDNCs.

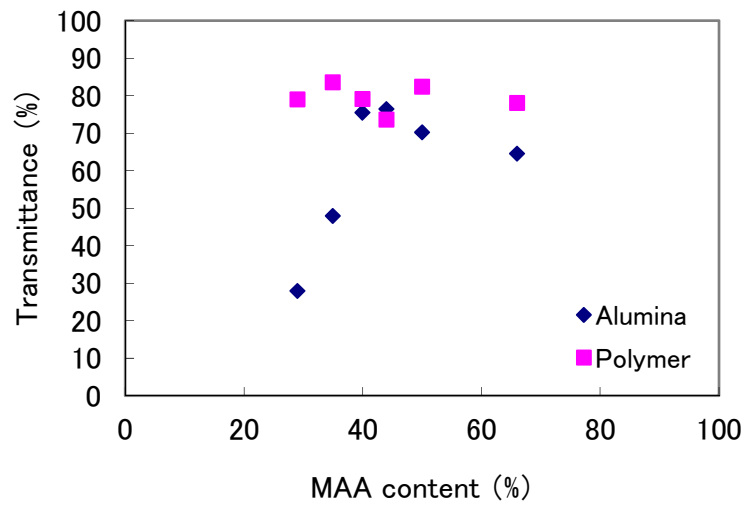

Figure 2. MAA content and transmittance of PDNC with different titania nanoparticle.

Transmittance changes with different molecular weights of binder polymers were plotted in Figure 3. The higher molecular weight polymer made the transparency lower, which means that high molecular weight polymer acts as flocculant.

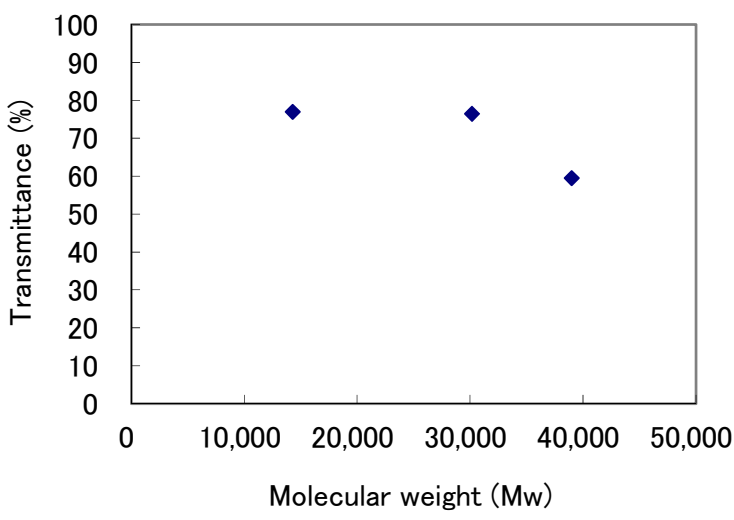

Figure 3. Molecular weight of binder polymer and transmittance of PDNC

Different kinds of solvents were used for diluting varnish. The kinds of solvents affected the transparency of PDNC. The relation of solubility parameter (SP) and transmittance was plotted in Figure 4. There is a tendency that the solvents with high SP increased the transparency of the nanocomposite. The highest transparency was obtained in the case of using DMF as a solvent.

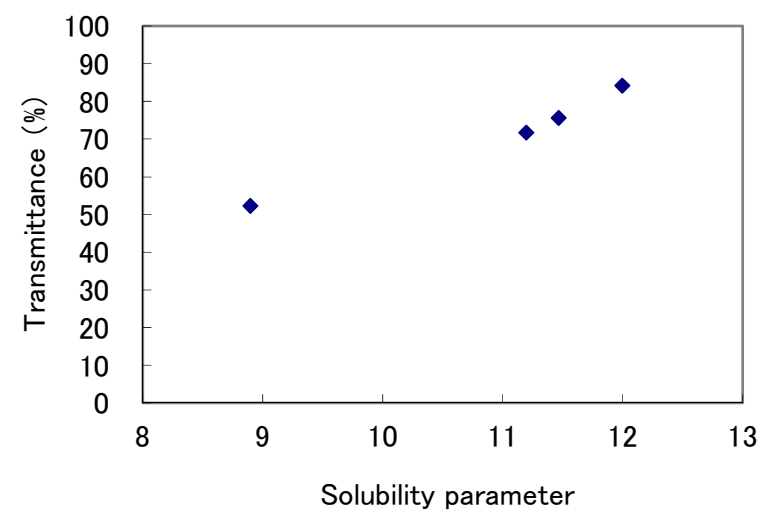

Figure 4. Solubility parameter of solvent and transmittance of PDNC.

To study the effect of solvents, we prepared the diluted raw nanoparticles by various solvents and measured particle sizes (Figure 5). We found that the transmittance was inversely proportional to the particle size. The fact indicates that the transparency of nanocomposite is determined by the particle size in a varnish state. The high SP solvent seems to stabilize the surface charge of nanoparticles to prevent aggregation. In the study of monomer composition, the higher MAA composition gave the higher PDNC transparency. This also seems that the binder polymer with higher MAA content has higher SP value. 


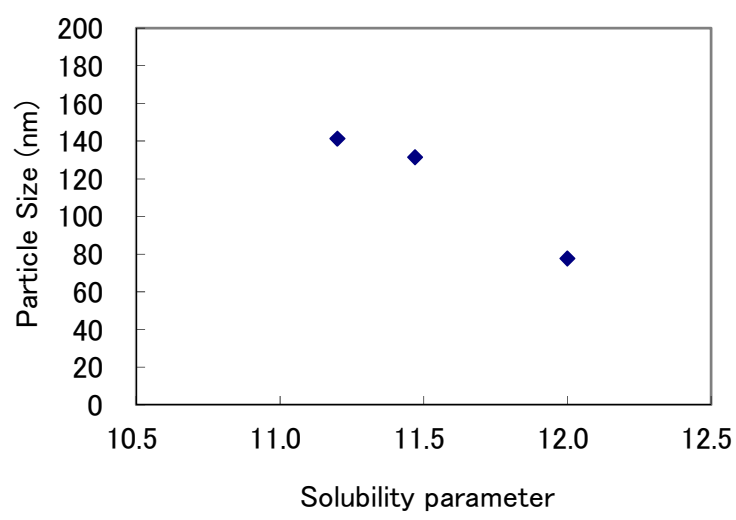

Figure 5. Solubility parameter of the solvent for PDNC and particle size in the diluted solution.

On the other hand, there is a possibility that a micro-phase separation of matrix induced an aggregation of the particles to change particle sizes and transmittances. In order to confirm that, we compared the atomic force micrographs (AFM) with or without nanoparticle to see the relation between the domain size and the particle size (Figure 6: without nanoparticle, Figure 7: with nanoparticle). It was found that the nanocomposite showed a uniform surface with well distributed nanoparticles, whereas the matrix without particle showed the phase separation with bicontinuous layer. There was no consistence between two images, showing that the particle size in the nanocomposite has no direct relation with phase separation.

From these considerations, we concluded that the transmittance of nanocomposite was determined by the dispersion state of nanoparticles in a varnish.

To sum up, as for the transparency of PDNC, it is important for the binder polymer to have (1) alternating monomer sequence, (2) low molecular weight, and (3) high SP.

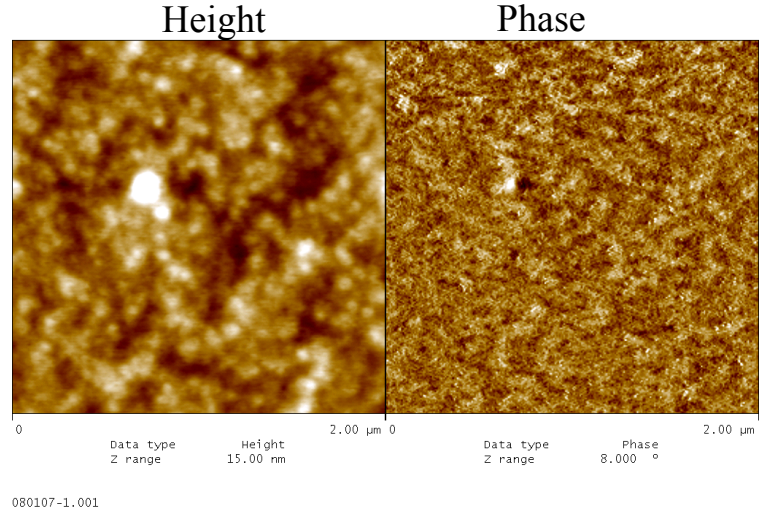

Figure 6. AFM images of a matrix without nanoparticle (binder polymer/ crosslinker).

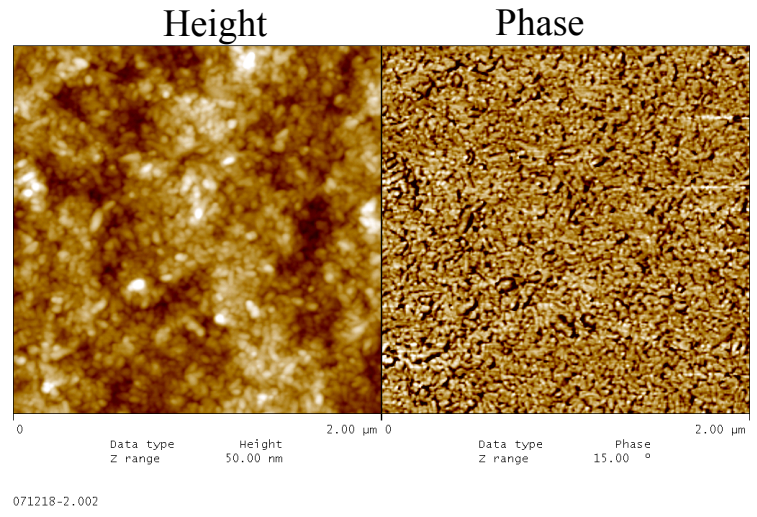

Figure 7. AFM images of a nanocomposite with nanoparticle (binder polymer/crosslinker/nanofiller).

3.2. Photolithographic property of PDNC and binder polymer composition

The photolithography of PDNCs was performed using three different compositions. Figure 8 shows the optical micrograph images of the resulting patterns. Comparing with monomer sequence in binder polymer, the alternating sequence has fewer residues than that of the random sequence. For polymer composition, the polymer with higher

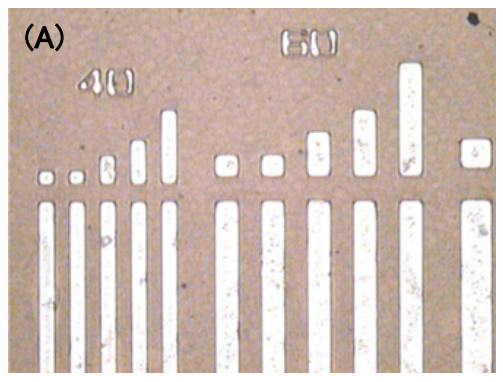

Random (St/MAA $=60 / 40$ )

Residue: a lot

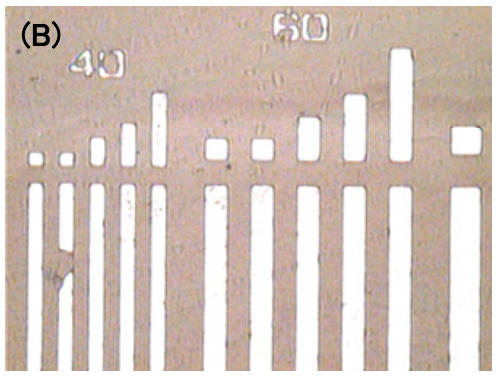

Alternating $(\mathrm{St} / \mathrm{MAA}=60 / 40)$

Residue: a few

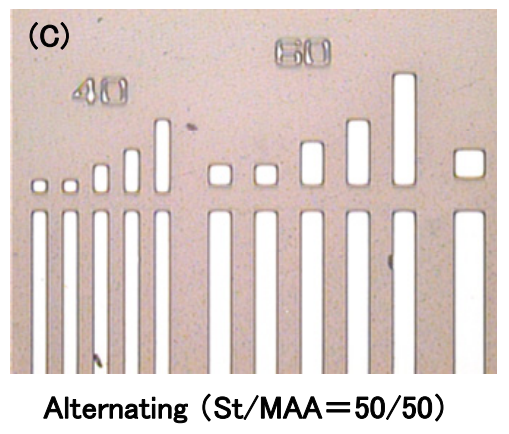

Residue: NO

Figure 8. Pictures of patterns 
MAA content reduced residues.

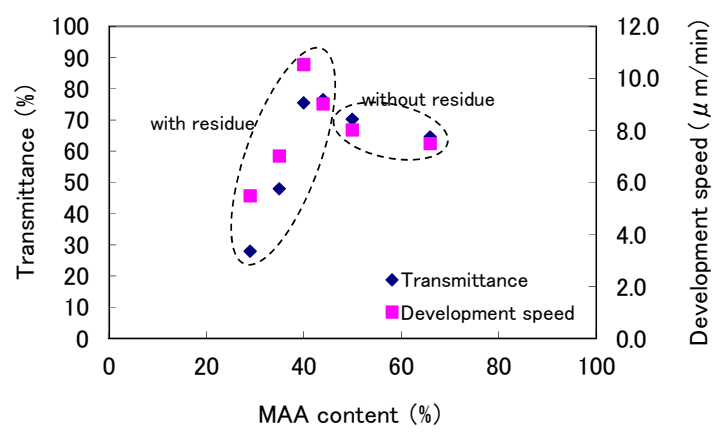

Figure 9. MAA content and transmittance of PDNC.

Development speeds of PDNCs with different MAA contents of binders were measured and overwritten in the data of Figure 2 (Figure 9). The transmittances and development speeds of PDNCs have a similar tendency. The development speed had a maximum with the binder polymer which gave the highest transmittance. No residue was observed with using the binder which had more than $50 \%$ of MAA content.

From the viewpoints of both transparency and patternability, it is desirable that an alternating sequence and a more than $50 \%$ of MAA content for the binder are both important for PDNCs.

We prepared four kinds of PDNCs with different titania content and measured the refractive index of each samples. The refractive index became higher with increasing titania content. The PDNC containing titania of $50 \mathrm{wt} \%$ showed the refractive index of 1.75 .

\section{Conclusion}

In this study, we examined the relationship of binder polymer compositions and lithographic properties and got following conclusions; (1) The alternating sequence of binder polymer is preferable to the nanocomposite of excellent transparency. (2) The moiety of methacrylic acid is necessary to be $50 \%$ or more for the residue-free patterning. The photo-defineable nano-composite containing alternating copolymer and nanotiania gives a patternable nanocomposite with high refractive index.

\section{Acknowledgement}

We thank Prof. J. Yin and Dr. X. Jiang of Shanghai Jiao Tong University for discussion and comments on this research. We also thank TEICA Corporation for supplying nanofiller.

\section{References}

1. Z.-K. Zhu, J. Yin, F. Cao, K. Shang, Q. Lu, Advanced Materials, 12(2000), 1055.

2. L. Li, L. Qinghua, W. Wenkai, Z. Zikung, W. Zonggang, Mater. Chem. Phys.,.74(2002), 210.

3. Z.-M. Liang, J. Yin, J-H. Wu, Z.-X. Qiu. F.-F. He, Eur. Polym. J., 40(2004), 307.

4. L..-C. Hsu, U. Wang, J.-S. King, J.-L. Jeng, Polymer, 44 (2003), 5533.

5. Y. Liu, L. Wang, C. Pan, Macromolecules, 32(1999), 8305.

6. Brandrup; Immergut; Grulke; Abe, Polymer Handbook (4th Edition), (2003). 